\title{
MEMS-based optical mini- and microspectrometers for the visible and infrared spectral range
}

\author{
R F Wolffenbuttel
}

Department for Microelectronics, Faculty of EEMCS, Delft University of Technology, Mekelweg 4, 2628 CD Delft, The Netherlands

Received 13 January 2005, in final form 18 April 2005

Published 20 June 2005

Online at stacks.iop.org/JMM/15/S145

\begin{abstract}
Miniaturized free-field based optical microspectrometers have huge potential for application in industry, science, medicine, agriculture and biology. State-of-the-art is the micro-assembly of micromachined optical components on a mini-bench and the trend is towards fully integrated optical microsystems. Complete silicon IC compatible MEMS-based opto-electrical microsystems on a single chip may offer huge cost benefits in these potentially high-volume applications. On-chip integration does, however, impose limitations. The required process compatibility and limited choice of acceptable materials does not necessarily give optimum optical performance. Also, the dimensional downscaling is not generally an optical advantage. This overview discusses grating-based and interferometer-based mini- and microspectrometers, shows performances already reported, the trends, the potential, the limitations and approaches to obtain a sufficient optical performance, in terms of spectral resolution and throughput, for serving the majority of applications.
\end{abstract}

(Some figures in this article are in colour only in the electronic version)

\section{Introduction}

Optical spectrometers are widely used for the highly sensitive and highly selective measurement of the composition of a gas or a fluid. The IR absorption spectrum is used to determine the composition of a gas [1] and in chemical chromatography for analysing the composition of a sample fluid [2]. The fluorescence spectrum is also used in chemistry to identify the composition and concentrations of dissolved ions [3, 4]. Fluorescence signals are also investigated for monitoring of photosynthesis in plants [5]. Miniaturized systems have been realized for monitoring the ozone in the earth atmosphere from a satellite [6].

Instruments conventionally used are typically bulky laboratory equipment with a performance that often exceeds by far what is required in industrial applications, where issues such as costs, sample volume and measurement time prevail. Miniaturized systems have been realized that are better suited for these applications; however, these still rely on micro-assembly and conventional fabrication technologies [7]. Optical microelectromechanical systems (MEMS) have been realized that satisfy these additional requirements better. These are small, lightweight and some are fabricated using silicon IC process compatible technologies, thus featuring the possibility for realizing an intelligent opto-electronic system by on-chip co-integration of optics and microelectronics [8]. However, the dimensional downscaling is poorly compatible with high-quality optics. Moreover, the in-system integration of components for optical signal conditioning (lenses or mirrors for collimation and focusing of incident and dispersed light respectively) is difficult to achieve, especially in the case of single-chip silicon optical systems with co-integrated optics and integrated circuits. This causes the primary performance parameter, the spectral resolution $(R=\lambda / \Delta \lambda$, where $\lambda$ denotes the wavelength tuned to and $\Delta \lambda$ the $-3 \mathrm{~dB}$ power bandwidth), to be limited to $R=100$ for minisystems based on the assembly of MEMS components and $R=20$ for integrated silicon microsystems [9]. 


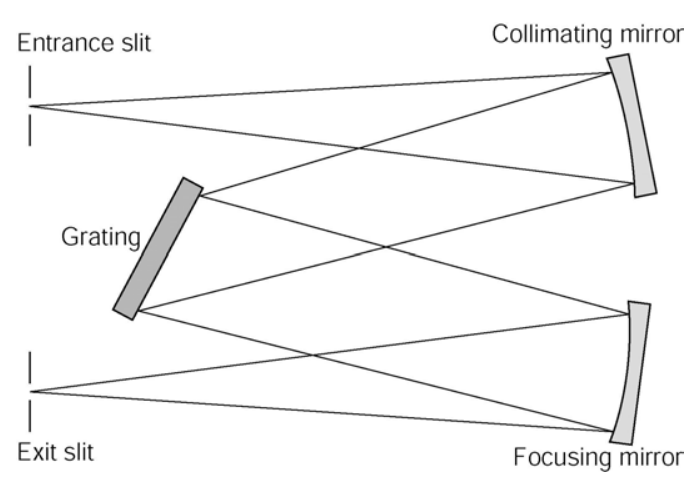

Figure 1. Simplified schematic of the grating-based spectrograph.

\section{Classification of spectrometers with potential for optical MEMS implementation}

The essential property of a free-field based optical system is the continuous (i.e. non-layered) propagation medium (usually air). The classification of the various spectrometer types is determined by the dispersion element or approach used and includes microspectrometers based on (a) prisms, (b) gratings and (c) interference of split components. Prism-based spectrometers are not suitable for miniaturization and are not considered here.

Grating-based microspectrometers use the interference of incident light at the grating for dispersion. The typical optical system is shown in figure 1. Light passes through a narrow entrance slit and is converted into a parallel beam using a collimating lens or mirror. Dispersion occurs at the grating and the dispersed spectrum is projected on the exit slit using a focusing lens or mirror. The spectrum is scanned across the exit slit by rotation of the focusing mirror or the grating. Grating-based microspectrometers have been realized for both the visible and near-infrared part of the spectrum using microfabrication techniques. As will be demonstrated, the performance is limited due to the use of a transmission grating and the omission of optical signal conditioning.

Interference-based microspectrometers involve splitting of the incident light beam into two parts and having these interfere after travelling over two optical paths with a welldefined difference in length. The path length difference relative to the wavelength is an effective approach for selecting a spectral component. Changing the path length difference in time can be used for a complete spectral scan of a well-defined part of the spectrum. There are a number of variations on this general operating principle and figure 2 shows the three most-widely used versions, which also have huge potential for microfabrication.

The Michelson interferometer uses one beam splitter and two mirrors (M1 and M2) to define the two optical paths. The difference in distance between the splitter and the mirrors defines the optical path length difference. Measuring the path length increase between the positions for two maxima or minima of the interference pattern detected at the detector by displacing one of the mirrors in the direction of the optical axis provides the measured wavelength of the incident light. The Mach-Zehnder uses two splitters and provides a better isolation between input and output. Both the Michelson and Mach-Zehnder interferometers are very suitable for

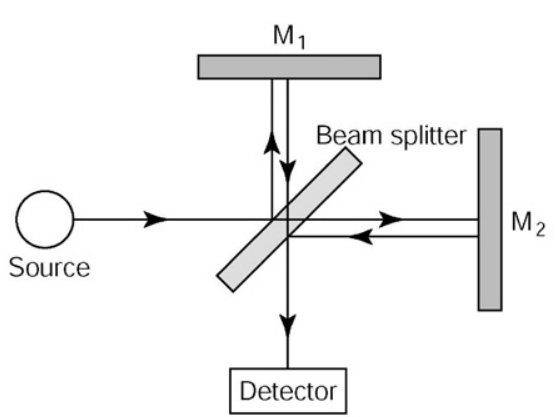

(a)
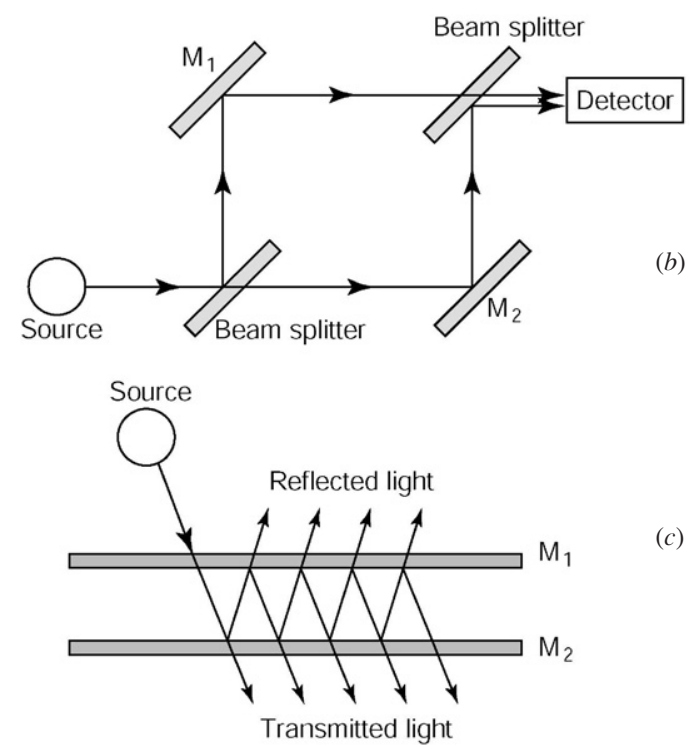

Figure 2. Operating principle of most-frequently used interferometer-based spectrometers: (a) Michelson, (b) Mach-Zehnder and (c) Fabry-Perot.

integration in a glass fibre or a waveguide, since the splitting and combining of light can be achieved relatively easily by the overlapping of fibres or waveguide channels. Moreover, the path length difference is defined by the difference in length between the fibres or the defined channels in the waveguide.

The beam splitter is more difficult to integrate in a freefield based MEMS microspectrometer and for that reason most systems reported about are based on the Fabry-Perot interferometer. The basic device is composed of two parallel mirrors with the reflecting surfaces facing each other and spaced a well-defined distance apart. Incident light is transmitted into the resonance cavity and is transmitted to the detector after multiple reflections at M1 and M2. Constructive interference takes place only for wavelengths that fit in the resonance cavity.

Magnetic, thermal or electrostatic actuation techniques available to MEMS are used for the displacement of one of the mirrors, as is schematically shown in figure 3 . This figure also introduces a fourth technique used in MEMS-based microspectrometers, the Fourier microspectrometer [10]. It should be noted that the Fourier microspectrometer is basically a modification of the Fabry-Perot type. The following sections give an outline of the various microspectrometers with an increasing degree of miniaturization or integration of optical and microelectronic components and comment on the specific advantages and disadvantages, design constraints and the typical performance. 

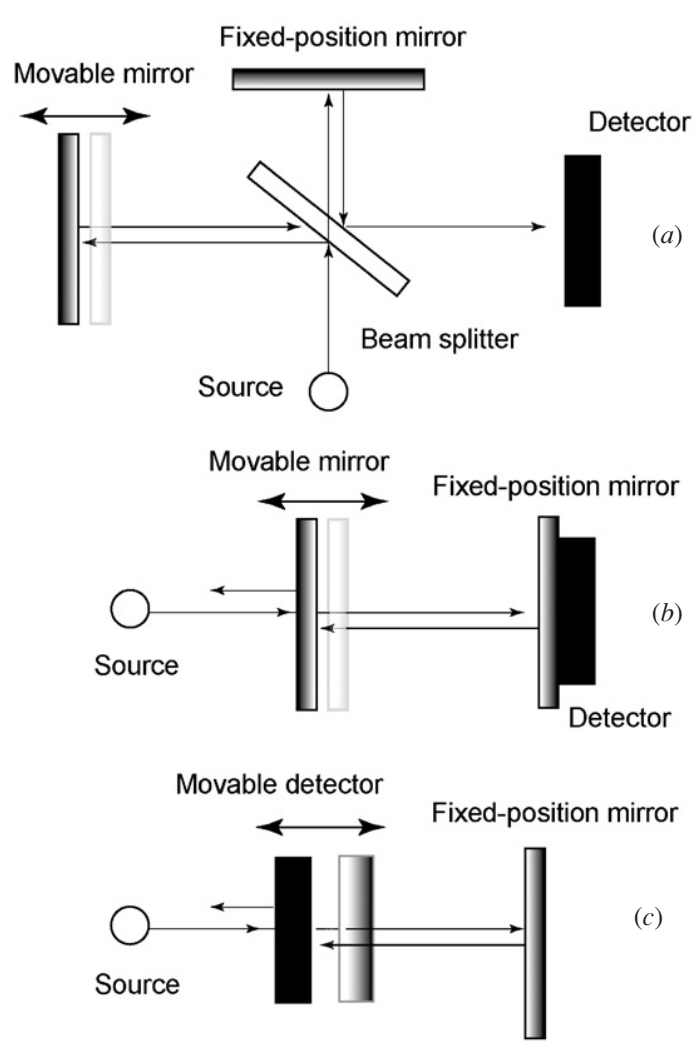

Figure 3. Scanning the spectrum in a interferometer-based microspectrometer: $(a)$ displacing a mirror in the Michelson interferometer, $(b)$ displacing one of the parallel mirrors in a Fabry-Perot resonator and $(c)$ displacing the detector in a Fourier microspectrometer.

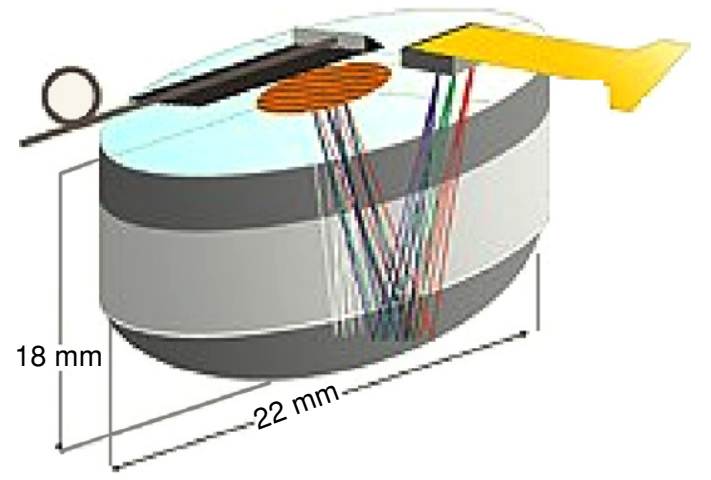

Figure 4. Schematic of a commercially available grating-based miniaturized spectrometer [11].

\section{Miniaturized spectrometers}

The most obvious approach is the microfabrication of the components followed by an assembly. Such microspectrometers have been made available from a Swiss company [11]. The device is shown in figure 4. The light is coupled to the entrance using a glass fibre. Replication techniques have been used for the fabrication of the grating and a commercially available photodiode array is used for the detector. The optical path is defined by the package. The spectral resolution is limited to $R=80$.

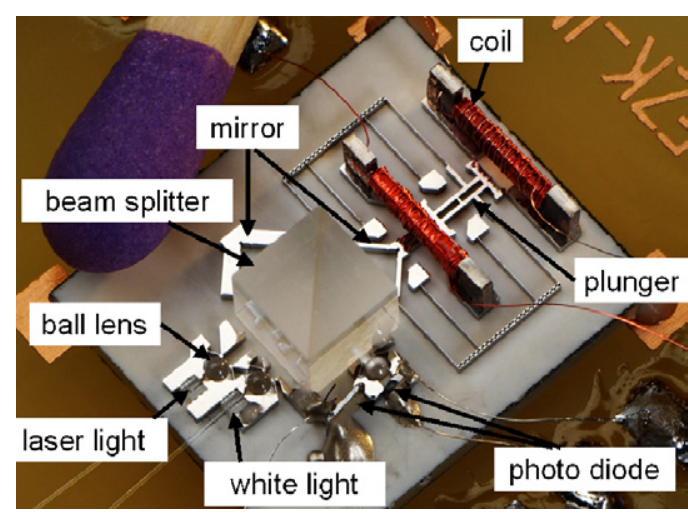

Figure 5. Michelson IR spectrometer on a NiFe optical mini-bench with LIGA-fabricated electromagnetic actuator for the displacement of the mirror [15].

Micromachined gratings have also been combined with a CCD camera in a hybrid system for operation as a spectrometer [12]. A resolution $R=70$ at $633 \mathrm{~nm}$ was reported. Liquid crystal technology has also been applied [13].

The next generation of miniaturized optical spectrometers involves the microfabrication of the optical components and the subsequent assembly on a micro-optical platform. A $14 \mathrm{~mm}$ long aluminium-nitride mini-bench with deformable aligner structures in nickel alloys fabricated in the LIGA process and silicon micromachined Fabry-Perot interferometer has been reported for use in the IR spectral range for application in the pharmaceutical industry [14]. Another IR spectrometer within this category is the Michelson interferometer with LIGA fabricated electromagnetic actuator for the displacement of the mirror required for scanning the spectrum $[15,16]$. The spectrometer is shown in figure 5 and includes an $11.5 \times 9.4 \mathrm{~mm}^{2}$ electroplated optical mini-bench of an electroplated soft $\mathrm{NiFe}$ alloy. The same material is used for the electromagnetic actuator, which enables a displacement of the mirror over $110 \mu \mathrm{m}$. A passively aligned silica ball lens is used for collimation and a InGaAs PIN photodiode for monitoring the fringe pattern. The detector limits the use to the near infrared and a resolution $R$ better than 60 at $1540 \mathrm{~nm}$ was reported.

Although silicon micromachining techniques are employed for the fabrication of optical components, these are combined in a system using micro-assembly. These microspectrometers are not directly silicon process compatible. High-volume applications are to be found in the areas of consumer products and quality inspection, which typically benefit from the extremely low cost in high-volume fabrication that silicon IC technology can offer. As a consequence, many research efforts have been reported in the literature on IC fabrication compatible integrated devices.

\section{MEMS-based microspectrometers in silicon}

\subsection{Grating type of microspectrometers}

Silicon bulk micromachining techniques have been employed for the fabrication of an integrated grating plus detector array in silicon for operation as a microspectrometer in the visible and infrared spectral range. The device intended for 


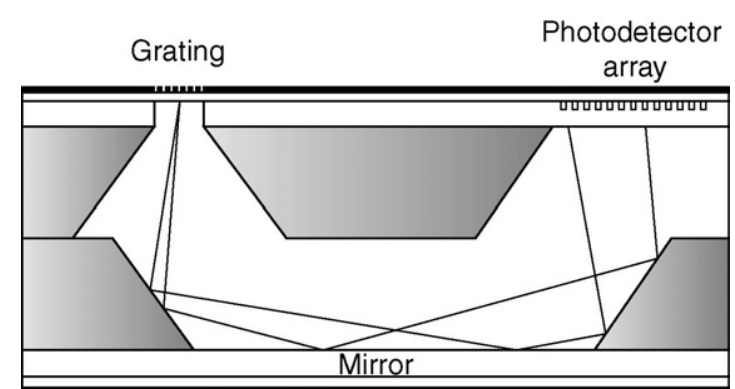

Figure 6. Schematic of a micromachined grating-based microspectrometer in silicon for operation in the visible part of the spectrum [17].

use in the visible spectral range is composed of two silicon p-type wafers with an n-epilayer on which electrochemically controlled etching (ECE) is applied to construct channels that form, after wafer bonding, an optical path between a grating and an array of photodiodes, as shown in figure 6 [17]. An electrically isolated region of epilayer is formed by deep boron diffusions to define the locations of the grating and backside-illuminated photodiodes. A 32-element $4 \mu \mathrm{m}$ pitch symmetrical ( $2 \mu \mathrm{m}$ line $/ 2 \mu \mathrm{m}$ space) transmission grating is used and $2 \mu \mathrm{m}$ wide photodiodes with $4 \mu \mathrm{m}$ pitch are formed by implantation into the epilayer. The aluminium, that is conventionally exclusively used for interconnect, is also used for fabrication of the grating and for shielding the array of photodiodes to prevent front-side illumination by the incident light. The light that is dispersed in the grating is projected onto a bevel that results from bulk micromachining on a $\langle 100\rangle$ wafer. After reflection on the epilayer of the lower wafer and a second bevel, the light is projected on the array of photodiodes.

The major problem in this device is the roughness of the bulk micromachined surfaces, which results in scattering of the reflected light. As the dispersed light is reflected three times before being projected on the photodiode array, a surface roughness comparable to that of the polished front side of a wafer is required. This problem was solved by avoiding the high-temperature steps that are used for epilayer growth and the deep boron diffusion, and by applying a BPSG reflow step [18]. The functional separation of the device into an active wafer with photodiodes and circuits and a passive reflection wafer, with low-temperature wafer bonding at the very last processing step circumvents any fabrication compatibility infringement and is a huge advantage of this two-wafer solution [19].

The grating should be designed for a gap half the pitch for maximum suppression of the second-order spectrum in order to maximize the free spectral range when using a detector array aligned with the first-order diffraction spectrum. Simply using an interconnect mask with a pattern of strips of half the pitch between lines in a standard process is not enough to achieve this. The standard etching procedure has been optimized for electrical constraints (avoiding short circuits) and includes an aluminium overetch. This indicates that even this highly compatible process step needs to be analysed critically. A slightly modified IC compatible metal etch yields an adequate suppression of the second-order spectrum while maintaining interconnect integrity.

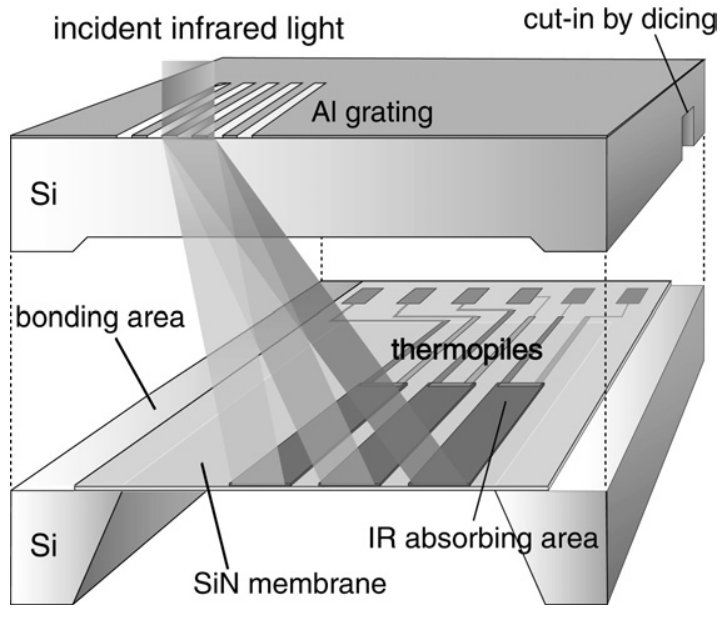

Figure 7. Schematic view of the grating-based microspectrometer for IR [20].

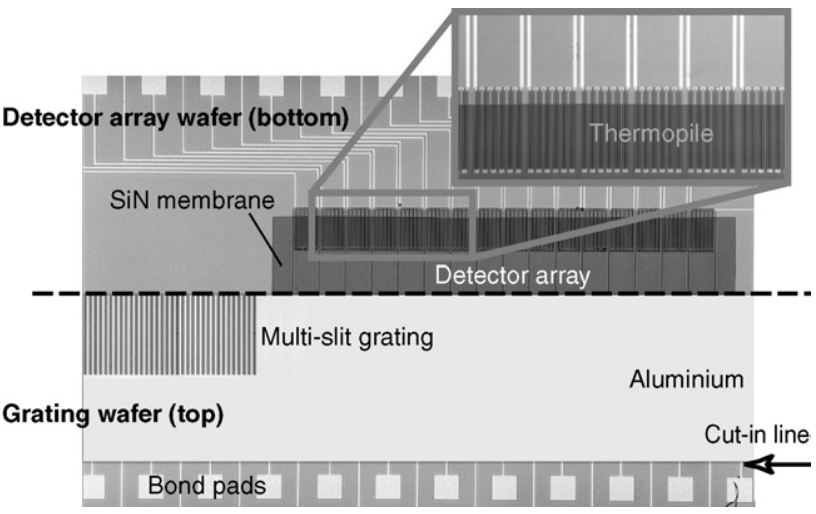

Figure 8. Microfabricated IR microspectrometer in silicon [20].

Microspectrometers have been developed for operation in various wavelength ranges in the IR spectral range. Bulk silicon and aluminium are used for the optical path and the multiple-slit grating, respectively. Silicon is highly transparent for wavelengths, exceeding $1 \mu \mathrm{m}$, beyond which free-carrier absorption can be disregarded. Therefore, the bulk silicon can be used to define the optical path rather than air, thus, overcoming the need to etch the light path. Also, in the IR spectral range, aluminium can be used for the fabrication of the grating. The IR spectrometer, shown schematically in figure 7, consists of two independently processed wafers, which are bonded in the final step [20]. Similar to the spectrometer operating in the visible spectral range, a twowafer approach is taken. However, thermal detectors are used due to the fact that silicon cannot be used for photon detection in the IR spectral range.

The grating is composed of 30 or 60 slits with a grating constant ranging from 4 to $20 \mu \mathrm{m}$. The length of the strips is $400 \mu \mathrm{m}$. The width of a single IR detector on the bottom wafer is $100 \mu \mathrm{m}$. The number of detectors in one array ranges between 6 and 16, and depends on the detectable wavelength range, which is in turn determined by the grating constant. Figure 8 shows the top view of a fabricated device. The upper half shows the detector array. The thermocouple responses at a $6 \mu \mathrm{m}$ grating constant for a number of monochromatic components are shown in 


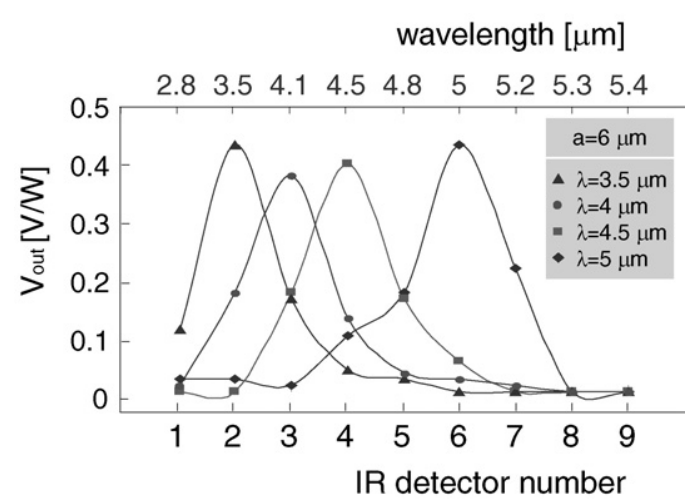

Figure 9. Spectral response of the IR microspectrometer with grating constant at $6 \mu \mathrm{m}$ when illuminated with a monochromator illuminator set at: $\lambda=3.5 \mu \mathrm{m}, 4 \mu \mathrm{m}, 4.5 \mu \mathrm{m}$ and $5 \mu \mathrm{m}$.

figure 9. The response peaks at a larger detector number in the case of longer wavelength illumination. The halfpower spectral resolution is $0.3-0.5 \mu \mathrm{m}$. It is interesting to note that thermocouples have been used without IR-absorbing coating. Basically, silicon oxide and silicon nitride layers have been used for this purpose. This approach circumvents any fabrication compatibility infringement. Since the response changes only by a factor 4 over a spectral range between $1 \mu \mathrm{m}$ and $8 \mu \mathrm{m}$, and spectrum is already dispersed, the correction for the wavelength-dependent absorption is determined by the detector position and can easily be implemented.

The spectral resolution in a grating-based spectrometer, when using the first-order diffraction spectrum, is in principle equal to the number of slits, $N$, in the grating: $R=N$ [21]. However, this assumes Fraunhofer rather than Fresnel diffraction. This can easily be assured in a system with optical signal conditioning; however, in a lensless system, it can be shown that the maximum useful number of slits of pitch $p$, where $p$ denotes the width of a grating element plus the space between two elements, is restricted by the wafer thickness, $t_{\mathrm{w}}$, according to: $N_{\max }^{2}=t_{\mathrm{w}} \lambda / p^{2}$. The pitch is limited by technological constraints and the diffraction angle that results from the maximum wavelength to be analysed. Using $p=$ $4 \mu \mathrm{m}$ and $\lambda=4 \mu \mathrm{m}$ yields: $N_{\max }=12$. The measured performance shown in figure 8 is in good agreement with this theoretical limit, however it also confirms a very limited performance. Optics for collimation and focusing is required for a sufficient spectral resolution for use in the majority of applications.

This overview is restricted to the state-of-the-art in freefield optics based microspectrometers. Hence, waveguidebased devices are not discussed here, despite their excellent performance in integrated optical chemosensors [22, 23]. Nevertheless, a hybrid system is included for comparison that combines waveguide technology and silicon micromachining, as shown in figure 10 [23]. The light to be analysed is introduced into the polymer waveguide through an optical fibre, is dispersed in a reflection grating that has been fabricated using deep reactive ion etching of the polymer and projected onto an array of fibres that guide the spectral components to an photodetector array. Device area is $18 \times 6.4 \mathrm{~mm}^{2}$ and the resolution obtained is $R=10$ over a spectral range extending between 720 and $900 \mathrm{~nm}$. It is interesting to note that this

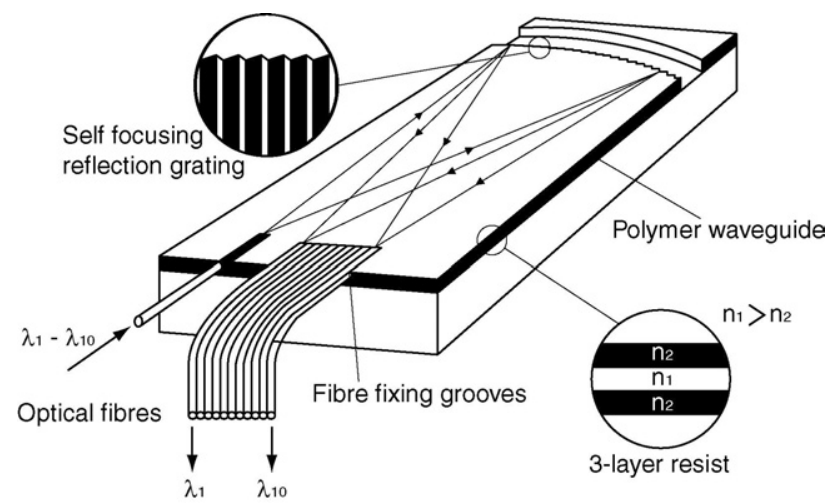

Figure 10. Waveguide-based spectrometer with fibres for in- and outcoupling of light. Reprinted from Sensors and Actuators, vol 25-27, Mohr J, Anderer B and Ehrfeld W, Fabrication of a planar grating spectograph by deep-etch lithography with synchrotron radiation, pp 571-5, Copyright (1991), with permission from Elsevier.

microspectrometer has so far been the only planar type that has become commercially available [24]. This approach has also been implemented using a silicon-oxi-nitride ( $\mathrm{SiON})$ layer as the waveguide. The properties of a waveguide enable the design of a self-focusing transmission grating. A FWHM of $9 \mathrm{~nm}$ has been reported at $450 \mathrm{~nm}$; thus, $R$ is about 50 [25].

\subsection{Interferometer type of microspectrometers}

The technological difficulty of integrating the beam splitter in silicon has concentrated the attention on the Fabry-Perot and Fourier interferometers, which are both basically composed of two highly reflective and parallel mirrors spaced a welldefined distance $d_{\mathrm{FP}}$ apart with the reflecting part facing each other. The space between the mirror surfaces is essentially a resonance cavity that contains standing waves of different modes (i.e. fitting an integer number of wavelengths). The operation is similar to the grating in the sense that a traversal leads to a phase difference $\varphi=2 \pi 2 n d_{\mathrm{FP}}$. Interference of these components results in a wavelength-dependent transmission described by the Airy transmission coefficient [26, 27, 28]. Resonance takes place at $2 n d_{\mathrm{FP}}=m \lambda_{0}$, with $m$ being an integer number. The spectral selectivity is expressed in terms of the finesse, $F$, of the resonator. In the case of two perfectly parallel mirrors, $F=\pi \sqrt{r} /(1-r)$, where $r$ denotes the amplitude reflection coefficient of the mirror.

The subtle difference between the Fabry-Perot and Fourier interferometer is that in the latter the detector is not covered by a mirror but is at a fixed position within the incident light path and measures the intensity a well-defined distance from a movable mirror (see figure 3). Displacement of the mirror enables the scanning of the standing wave that results from the interference at the detector position. This technique is therefore also referred to as the standing wave scanning spectrometer. As is explained in this section, the respective practical limitations cause the two types to be similar.

The simplest realization of the Fabry-Perot-based microspectrometer uses bulk micromachining on two wafers with subsequent wafer-to-wafer bonding [29-35]. Figure 11 shows the basic device structure. Applying a voltage makes it possible to tune the resonance cavity width to the desired 


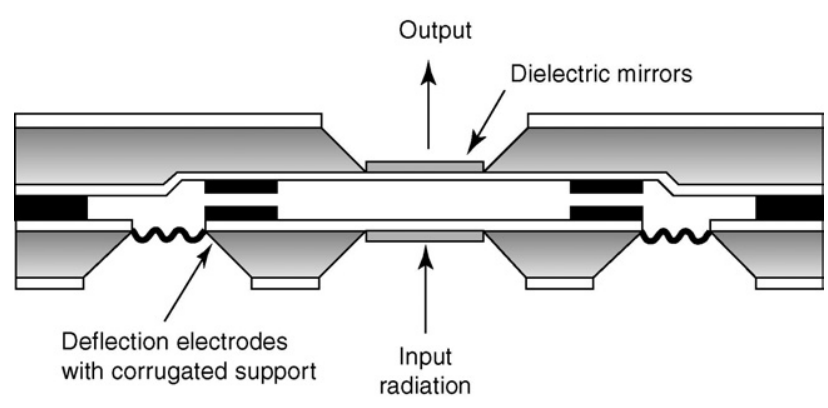

Figure 11. Microfabricated Fabry-Perot-based microspectrometer in silicon [29].

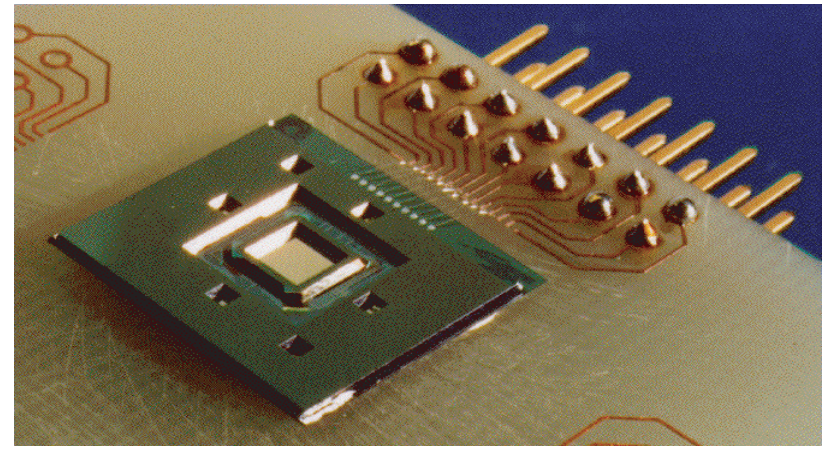

Figure 12. Photograph of the two-wafer bulk micromachined Fabry-Perot interferometer. A frame is used to keep the membrane flat [33].

wavelength. In an improved version, a silicon frame is used to ensure a flat membrane at the mirror area. The deformation of the membrane due to electrostatic force acting on it is in the membrane area outside the frame [33]. This technique prevents a reduced optical performance that results from the curvature of a simple suspended membrane with a mirror surface, at the expense of a higher voltage required for yielding a certain deflection at the same membrane area. Beam suspension [32] and corrugations [35] are also used for this purpose. A fabricated microspectrometer is shown in figure 12 .

The main design challenges of this type of Fabry-Perot interferometer are:

- the high voltage required to tune the movable mirror over a sufficiently large spectral range,

- the fabrication of mirror surfaces of sufficient reflectivity and flatness and

- achieving parallelism between the two mirrors.

An alternative approach that circumvents these problems uses 16 Fabry-Perot resonators of different fixed cavity spacings. Oxide layers are used to space the mirrors, thus ensuring parallel mirrors. Four subsequent masked oxide etch steps are applied to fabricate cavities of 16 different thicknesses. The 16 channels are designed to cover the entire visible spectral range. The fabrication of these Fabry-Perot resonators is compatible with a standard CMOS process, thus enabling the on-chip integration of circuits for selection and readout of the array photodiodes covered by the different resonators. The resulting device is shown in figure 13 [36].

An additional channel is used for the compensation of dark current and the scattering, which is due to roughness of

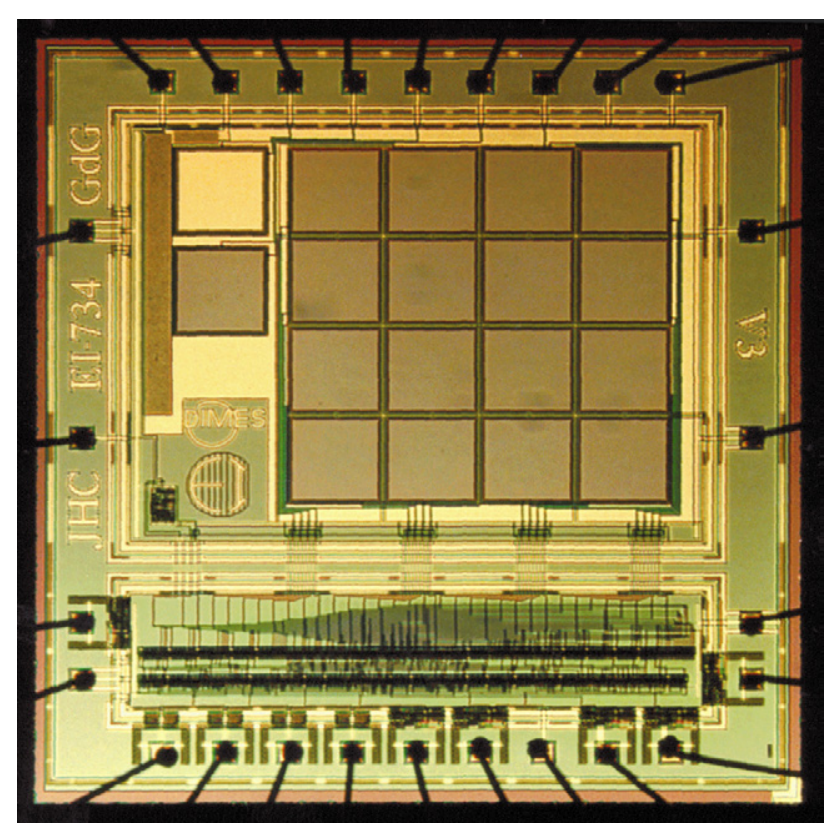

Figure 13. CMOS multichannel microspectrometer with 16 Fabry-Perot resonance elements on top of a chip containing a photodiode array and circuits for readout and interfacing a serial bus [36].

the mirror material [37]. A disadvantage of this approach is that it is very wasteful with impinging optical power. The light is projected, and thus distributed over the array before dispersion; thus, each etalon receives only the part of the power proportional to the number of elements. The resolution is intrinsically limited to $R=16$; however, measurement results reported indicate that it is even further reduced to $R=8-10$ [37]. The limited performance is due to the fact that Fabry-Perot resonators in a microspectrometer are usually operated in the first-order mode $(m=1)$ with the halfpower bandwidth $(\mathrm{FWHM}=\Delta \lambda)$ equal to the finesse $F_{\text {int }}=$ $\pi \sqrt{r} /(1-r)$. The finesse is limited by the reflectivity of the material used for fabrication of the mirror. When using thin-film metals for IC compatibility, a designer is limited by the film thickness, which is constrained by reflectivity and absorption. High resolution requires high reflectance of the mirrors and high throughput implies low absorption [38]. In aluminium, reflection increases from $10 \%$ to $85 \%$, while transmission decreases from $90 \%$ to $5 \%$ when increasing film thickness from $5 \mathrm{~nm}$ to $10 \mathrm{~nm}$ [39]. These data indicate that absorption increases rapidly with metal layer thickness, thus reducing throughput at resonance and consequently leading to a poor signal-to-noise ratio. Throughput in practical devices typically peaks at resonance to $10 \%$. High reflectivity and low absorption can be combined in a multiple-layer dielectric mirrors [40]; however, the fabrication of such a stack of alternating materials is marginally compatible with silicon microelectronic processes.

The spectral resolution is further limited by the parallelism of the two mirrors. Changes in the cavity width within one device obviously yields a range of resonance frequencies and, thus, of a widening of the overall resonance. This effect can be analytically expressed in the effective finesse: $1 / F_{\text {eff }}^{2}=1 / F_{\text {int }}^{2}+1 / F_{\mathrm{D}}^{2}$, where $F_{\text {int }}$ denotes the intrinsic finesse discussed before and only depending on the material 


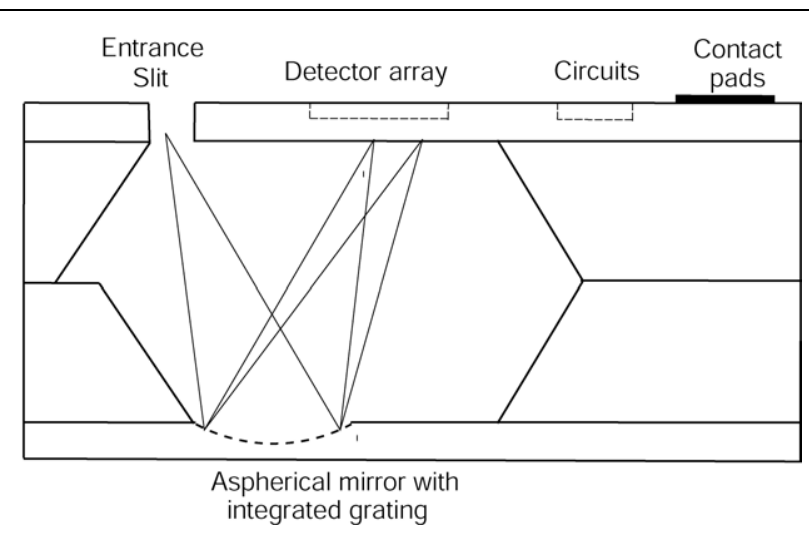

Figure 14. Concept for an enhanced-resolution grating-based microspectrometer.

property $r$, and $F_{\mathrm{D}}$ denotes the defect finesse depending on system defects such as limited parallelism [34, 35]. Multiple location servo control of cavity spacing based on capacitive displacement sensing and electrostatic actuation has been applied to optimize parallelism [41]; however, $F_{\text {eff }}$ in micromachined silicon devices is typically limited to $F_{\text {eff }}=20$.

Another issue is the free spectral range (FSR), which is in principle limited to avoid overlap between the shortest wavelength in the first-order mode and the longest wavelength is the subsequent second-order mode: FSR $=\lambda^{2} / 2 d_{\mathrm{FP}}$. However, practical cavity width modulation using electrostatic actuation limits $\Delta d_{\mathrm{FP}} / d_{\mathrm{FP}}$ to about $30 \%$ because of the pull-in of the microstructure.

Finally, the fabrication of IC compatible wideband microinterferometers is also complicated by the fact that thinfilm metals exhibit a wavelength dependence of the reflectivity. For UV operation, $\mathrm{Al}$ is to be used, whereas $\mathrm{Au}$ is the material of choice for mirrors operating in the IR spectral range $[38,39]$.

The advantage of the Fourier-based interferometer, as compared to the Fabry-Perot based device, is that the reflecting mirror can be optimized for reflectivity and the film thickness is not critical. The disadvantage is the absorption in the detector. This absorption should, on the one hand, be minimum to achieve full constructive and destructive interferences. On the other hand, absorption is needed to yield a detector signal. A compromise is inevitable. Consequently, the practical detector is a (poor) absorber and reflector, which is comparable to the practical Fabry-Perot device discussed. A resolution $R=100$ at $633 \mathrm{~nm}$ was reported [10]. The detector is generally based on amorphous silicon on a glass substrate $[42,43]$.

\section{Conclusions}

MEMS-based microspectrometers in silicon have been reported in the literature. Both grating-based and optical resonance cavity based devices have been investigated. The primary performance parameter, the spectral resolution, has been limited to $R=10-20$. Grating-based devices are typically without a lens or deformed mirror to focus the dispersed spectrum onto a detector array, which yields a simple system, but also imposes fundamental constraints that lead to this relatively poor optical performance. The spectral resolution of Fabry-Perot-based microspectrometers operated in the first-order mode is limited to a similar value. The enhanced mode of operation that is theoretically available when using a higher-order mode is difficult to realize in a practical microspectrometer due to imperfect parallelism, and absorption and reflection of the materials applied for fabrication of the mirrors that form the optical resonator.

The essential conclusions to be drawn from this outline are that the dimensional downscaling that benefits mainstream microelectronics is not an undisputed advantage in lensless MEMS-based microspectrometers. As a consequence, future work should be directed towards the integration of optical signal conditioning in an IC-compatible fashion. One possible concept for a higher-performance silicon process compatible microspectrometer is shown in figure 14. Deformable mirrors can be used for collimating and focusing [44]. Other essential optical components, such as tuneable entrance slits, have been reported in the literature [45]. Spacers may be included between the wafers for implementation of a very different compromise between technology and performance. Many applications can be served in the case of a spectral resolution exceeding $R=1000$ while maintaining the dimensional advantages. LIGA remains a highly competitive assembly technology in demanding applications in case IC-compatibility constraints prove too restrictive [46].

Finally, it should be noted that signal-processing techniques are available to improve the characteristics [47]. Integrated microspectrometers offer the opportunity to integrate spectrometer, readout and signal processing in a genuine micro-device.

\section{References}

[1] Stuart B 1996 Modern IR Spectroscopy (New York: Wiley)

[2] Iordanov V, Lubking G, Ishihara R, Wolffenbuttel R F, Sarro P M and Vellekoop M J 2002 Silicon thin-film UV filter for NADH analysis Sensors Actuators A 97-98 161-6

[3] Rossberg D 1995 Silicon micromachined infrared sensor with tuneable wavelength selectivity for application in infrared spectroscopy Sensors Actuators A 46-47 413-6

[4] Minas G, Martins J S, Pereira C, Lima C, Wolffenbuttel R F and Correia J H 2004 Biological microsystems for measuring uric acid in biological fluids Sensors Actuators A $11033-8$

[5] Rosema A 2002 Potential of chlorophyll fluorescence for remote sensing of canopy photosynthesis Proc. OECD Workshop on Remote Sensing for Agriculture for the Environment (Kiffisia, Greece, 17-20 Sept. 2002)

[6] Smorenburg C, Draaisma F, Oprel P, te Plate M, Visser H and de Winter D 2001 OMI-EOS: calibration stimuli for OMI at TNO TPD Proc 52nd Int. Astronautical Congress (Toulouse, France, 1-5 Oct. 2001)

[7] Smith J P 2000 Spectrometers get small Anal. Chem. 72 $853 \mathrm{~A}-8 \mathrm{~A}$

[8] Wolffenbuttel R F 2001 Silicon photodetectors with a selective spectral response Sensors Update vol 9 ed H Baltes, J Hesse and J Korvink (New York: Wiley) pp 69-101

[9] Wolffenbuttel R F 2004 State-of-the-art in integrated optical microspectrometers IEEE Trans. Instrum. Meas. $\mathbf{5 3}$ 197-202

[10] Manzardo O, Herzig H P, Marxer C R and de Rooij N F 1999 Miniaturized time-scanning Fourier transform spectrometer based on silicon technology Opt. Lett. 24 1705-7

[11] www.spectrosolutions.ch 
[12] Yee G M, Maluf N I, Hing P A, Albin M and Kovacs G T A 1997 Miniature spectrometers for biochemical analysis Sensors Actuators A $\mathbf{5 8}$ 61-6

[13] Hirabayashi K, Tsuda H and Kurokawa T 1991 Narrow-band tunable wavelength selective filters for Fabry-Perot interferometers with a liquid crystal intercavity IEEE Photon. Technol. Lett. 3 213-5

[14] Kotidis P, Atia W, Kuznetsov M, Fawcett S, Nislick D, Crocombe R and Flanders D C 2003 Optical, tunable filter-based micro-instrumentation for industrial applications Proc. Expo www.isa.org

[15] Wallrabe U, Mohr J and Solf C 2004 Mini-FT spectrometer for the near-infrared Proc Eurosensors XVIII (Rome, Italy, 12-15 Sept. 2004) pp 160-1

[16] Mohr J A, Last A, Hollenbach U, Oka T and Walrabe U 2003 A modular fabrication concept for microoptical systems J. Lightwave Technol. 21 643-7

[17] Kwa T A and Wolffenbuttel R F 1992 Integrated grating/detector array fabricated in silicon using micromachining techniques Sensors Actuators A 31 259-66

[18] Kwa T A, French P J, Wolffenbuttel R F, Sarro P M, Hellemans L and Snauwaert J 1995 Anisotropically etched silicon mirrors for optical sensor applications J. Electrochem. Soc. 142 1226-33

[19] Wolffenbuttel R F (ed) 1996 Silicon Sensors and Circuits: On-Chip Compatibility (London: Chapman \& Hall)

[20] Kong S-H, Wijngaards D D L and Wolffenbuttel R F 2001 Infrared microspectrometer based on a diffraction grating Sensors Actuators A 92 88-95

[21] Goldman D, White P and Anheier N 1990 Miniaturised spectrometer employing planar waveguides and grating couplers for chemical analysis Appl. Opt. 29 4583-9

[22] Heideman R G and Lambeck P V 1999 Remote opto-chemical sensing with extreme sensitivity: design, fabrication and performance of a pigtailed integrated optical phase modulated Mach-Zehnder interferometer system Sensors Actuators B 61 100-27

[23] Mohr J, Anderer B and Ehrfeld W 1991 Fabrication of a planar grating spectrograph by deep-etch lithography with synchrotron radiation Sensors Actuators A 25-27 $571-5$

[24] www.getspec.com (select NIR1750)

[25] Sander D and Müller J 2001 Self-focusing phase transmission grating for an integrated optical spectrometer Sensors Actuators A 88 1-9

[26] Born M and Wolf E 1975 Principles of Optics 5th edn (New York: Pergamon)

[27] Saleh B and Teich M C 1991 Fundamentals of Photonics (New York: Wiley)

[28] Vaughan J M 1989 The Fabry-Perot Interferometer: History, Theory, Practice and Applications (Bristol: Hilger)

[29] Raley N F, Ciarlo D R, Koo J C, Beiriger B, Trujillo J, Yu C, Loomis G and Chow R 1992 A Fabry-Perot microinterferometer for visible wavelengths Proc IEEE Workshop on MEMS (Travemünde, Germany, 4-7 Feb. 1992) pp 170-3
[30] Tran A T T D, Lo Y H, Zhu Z H, Haronian D and Mozdy E 1996 Surface-micromachined Fabry-Perot tunable filter IEEE Photon. Technol. Lett. 8 393-5

[31] Zavracky P M, Denis K L, Xie H K, Webster T and Kelley P A 1998 Micromachined scanning Fabry-Perot interferometer Proc SPIE 3514 179-89

[32] Aratani K, French P J, Sarro P M, Poenar D P and Wolffenbuttel R F 1994 Surface micromachined tuneable interferometer array Sensors Actuators A 41-42 17-23

[33] Correia J H, Bartek M and Wolffenbuttel R F 1999 Bulk micromachined tunable Fabry-Perot micro-interferometer for the visible spectral range Sensors Actuators A 76 191-6

[34] Correia J H, Bartek M and Wolffenbuttel R F 2000 High-selectivity single-chip spectrometer in silicon for operation in the visible part of the spectrum IEEE Trans. Electron Devices 47 553-9

[35] Jerman J H, Clift D J and Mallinson S R 1991 A miniature Fabry-Perot interferometer with a corrugated silicon diaphram support Sensors Actuators A 29 151-8

[36] Correia J H, de Graaf G, Kong S-H, Bartek M and Wolffenbuttel R F 2000 Single-chip CMOS optical micro-interferometer Sensors Actuators A 82 191-7

[37] Correia J H, de Graaf G, Bartek M and Wolffenbuttel R F 2001 A CMOS optical microspectrometer with light-to-frequency converter, bus interface and stray-light compensation IEEE Trans. Instrum. Meas. 50 1530-7

[38] Ordal M A 1983 Optical properties of the metals $\mathrm{Al}, \mathrm{Co}, \mathrm{Cu}$, $\mathrm{Au}, \mathrm{Fe}, \mathrm{Pb}, \mathrm{Ni}, \mathrm{Pd}, \mathrm{Pt}, \mathrm{Ag}$, Ti and $\mathrm{W}$ in the infrared and far infrared Appl. Opt. 22 1099-119

[39] Rossberg D 1996 Optical properties of the integrated infrared sensor Sensors Actuators A 54 793-7

[40] Macleod H A 1986 Thin-Film Optical Filters 2nd edn (Bristol: Hilger)

[41] Hicks T R, Raey N K and Atherton P D 1984 The application of capacitance micrometry to the control of Fabry-Perot etalons J. Phys. E: Sci. Instrum. 17 49-54

[42] Kung H L, Bhalotra S R, Mansell J D, Miller D A B and Harris J S 2002 Standing wave transform spectrometer based on integrated MEMS mirror and thin-film photodetector IEEE Sel. Top. Quantum Electron. 898

[43] Stiebig H, Büchner H-J, Bunte E, Mandryka V, Knipp D and Jäger G 2003 Standing wave detection by thin-transparent $\mathrm{n}-\mathrm{i}-\mathrm{p}$ diodes of amorphous silicon Thin Solid Films $\mathbf{4 2 7}$ $152-6$

[44] Vdovin G V, Akhzar-Mmehr O, Sarro P M, de Lima Monteiro D W and Loktev M 2003 Arrays of spherical micromirrors and molded microlenses fabricated with bulk Si micromachining Proc. SPIE 4945 107-11

[45] Riesenberg R and Seifert T 1999 Design of spatial light modulator microdevices-micro slit arrays Proc. SPIE 3680 406-14

[46] Wallrabe U and Mohr J 2003 Modular microoptical system for sensors and telecommunication Sensors Update vol 11 ed H Baltes, J Hesse and J Korvinck (New York: Wiley) pp 144-74

[47] Bhalotra S R, Kung H L, Jiao Y and Miller D A B 2002 Adaptive time domain filtering for real-time spectral discrimination Opt. Lett. 27 1147-9 